\title{
Usage of RC index as a Good Representation for Characterising Rapid Variation Signals in Geomagnetic Field Studies.
}

\author{
Elvis Onovughe \\ Department of Earth Sciences, Federal University of Petroleum Resources, Effurun, \\ Nigeria
}

E mail (onovughe.elvis@fupre.edu.ng).

Accepted: 12 March 2018

\begin{abstract}
We present a study of the RC index as a better representation for characterising rapid variations globally than the Dst index. Using the CM4 model and modifying certain parts of the model allowing us to subtract specific field contributions, we generated the geomagnetic field measurements of interest which we use in calculating the eigenvalues and eigenvectors for combined observatory locations to study the rapid variations seen in our data for days away from quiet time. Result of the eigenanalysis show that the covariance comes between $X$ and $Y$ components of our combined geomagnetic observatory measurements, unlike what obtains in slow and longer variations in single observatory where the covariance is observed between $X$ and $Z$ residuals of the geomagnetic field. Also, the $X$ component of our combined observatory measurement residuals correlates very well with that of the RC index, matching the geomagnetic observatory rapid variation signals of our combined observatories. This good correlation between the $X$ component residuals of our combined observatory measurements and that of the RC index is further confirmed through high cross-correlation coefficients between their residuals which ranges from 0.70 to 0.90 in all the combined observatories studied globally. This suggests that the rapid variation is coming from a large-scale source, possibly the magnetospheric ring current, and the RC index as a good representation for rapid variation globally.
\end{abstract}

(c) 2018 BBSCS RN SWS. All rights reserved

Keywords: RC index, Geomagnetic Field

\section{Introduction}

The geomagnetic field is slightly asymmetrical in space and time, consisting of the relatively slowly varying main field (core or internal) source in the Earth's outer core, the nearly stable contributions from the upper lithosphere (crustal or terrestrial), and the more rapidly changing external (extraterrestrial) field sources. While the extraterrestrial field sources includes components that represent less than ten percent $1<$ $10 \%)$ of the total field measured at the Earth's surface, over ninety percent ( $>90 \%$ ) is produced by a selfsustaining geodynamo in the fluid core and can be explained by a tilted axial dipole. In addition to the temporally static crustal field from permanently magnetized rocks, the main field is also overlain by the comparably weak but highly variable induced fields in the electrically conducting parts of the upper mantle and crust. These weak and highly variable fields originate as secondary contributions from the rapidly changing geomagnetic fields generated by current systems in the magnetosphere and ionosphere.

Over all characterisation of the geomagnetic field has improved vastly over the last few decades occasioned by several low-orbiting geomagnetic satellites - Orsted, CHAMP, Sac-C and now, Swarm, coupled with the setting up of numerous permanent and temporary geomagnetic observatory stations all over the globe. This has not only led to much improved quality measurements of the geomagnetic field, it has also led to better techniques and strategies for separating the external field sources from the internal field sources, some of which have been investigated since the time of Gauss in 1839, when he applied Spherical Harmonic Analysis (SHA) to his observations of the magnetic vector field (Olsen et al. 2010). In order to analyse the short-term time series with regard to the current systems that regularly contribute to the external field signals recorded in observatory measurements, most internal contributions need to be eliminated from the measurements.

There are different geomagnetic field models i.e. both internal magnetic field and external magnetic field models, used for describing the field generated by different sources of the geomagnetic field. For example, most current internal magnetic field models describe the field generated in the magnetosphere using:

- Slowly varying spherical harmonic (SH) degree 1 or 2 external fields.

- Rapid Dst dependent fields.

Also, most current external field models include $\mathrm{SH}$ degree 1 or $\mathrm{SH}$ degree 2, and the rapid variations of the field are modelled using Dst index (Langel and Estes, 1985). The Dst index (Sugiura, 1964), which model the external geomagnetic field, particularly the rapid variation, combine the degree of the main field depression recorded at four mid-latitude observatory stations (Honolulu, HON; San Juan, SJG; Hermanus, HER; and Kakioka, KAK) to characterise the global strength of the magnetospheric disturbance field. See figure 1 for location of the four mid-latitude observatories used for calculating the Dst index.

Several researchers have performed different operations on the Dst index to try to enhance its use for geomagnetic field modelling. For example, Olsen et al. (2005), and Maus and Weidelt (2004) had it broken down into direct external (Est) and an indirect induced (Ist) signal. Mursula et al. (2011) reconstructed Dst for the years 1932-2002 and corrected for semi-annual and seasonal variations, a normalization according to observatory latitude and an unequal weighting of the stations in series of publications between 2005 and 2011 (see Karinen and Mursula 2006). They called the outcome of these changes Dcx (Mursula and Karinen, 2005). Additionally, Gannon and Love (2011) updated 
the removal of the solar-quiet variation $(\mathrm{Sq})$ and increased the temporal resolution of Dst to one minute. Despite all of these various improvements or attempts at enhancing Dst modelling abilities, it still suffers from baseline instabilities (Olsen et al. 2005) that limits its effectiveness for long and short term studies. As a result Olsen (2002) built the ring current (RC) index to improve time dependence.

In this study the CM4 model is used extensively as a comparison for our observatory measurements. The CM4 model (Sabaka et al. 2004) has a much more complex time dependence but the rapid variations of the fields are controlled by the Dst index. The model is capable of modelling some of the toroidal magnetic fields in the ionosphere, the effect of the ring current in the magnetosphere, and the effect of increased solar flux amongst other things. CM4 is constrained by the actual data recorded at observatories and during satellite missions, and can be used to gain understanding of how the field is behaving over short time scales. When this is tied to the behaviour of the field due to solar influences, CM4 can be used in extending expected field behaviours to locations where there is poor data coverage, based on the behaviour at locations where observations exist. This has been proposed to hopefully lead to improvement in remote referencing i.e. if a model (in this case CM4) measurement accurately represents the field behaviour over survey-scale time periods, then it could be used as a base station measurement for surveys where actual stations are far away or unavailable for whatever reasons. The CM4 model have performed creditably well during quiet periods, but have not done too well during periods of rapid variations for days away from quiet time period. The reason has been adduced to the external field description (Dst and F10.7) included in the CM4 model (Onovughe and Holme, 2015). In this study we have compared combined observatories at one time, for days away from quiet time, using CM4 model and the RC index. This is in a view to characterise the RC index as a good representation of all observatories for rapid variation studies. Assumption is that if it is a good representation, then this tells us that the rapid variations are coming from a large scale, and therefore corrections for days away from quiet times (i.e. exploration corrections) can be considered to be global. Also, observatory measurement results combined (more than a single base station) for days away from quiet time might be a good remote referencing technique.

\section{Data and Model}

\subsection{Data}

The measurements (data) used in this study are based on observatory hourly means (OHMs). The observatory measurements are extracted from measurement holdings available at the International Real-time Magnetic Observatory Network, INTERMAGNET (http://www.intermagnet.org). The network also provides information on observatory locations, possibly relocations and general measurement issues. The basic measurements obtained from the geomagnetic observatories in the INTERMAGNET network are hourly mean values of the three mutually orthogonal components - the North $(X)$, the East (Y), and the vertical downwards (Z) components. We checked the availability, quality and continuity of the observatory measurement over the studied period. Our hourly means were recorded only if more than one minute values are available, thus ensuring the reliability of our final hourly means measurements. The measurement set consists of hourly means computed from the available continued measurements at geomagnetic observatories between May 2003 and September 2006. All the measurements are daily variation measurements within a 24 hour period for selected days away from quiet time i.e. moderately disturbed days ( $\mathrm{Kp} \leq 5)$. In all measurements were obtained from 109 worldwide geomagnetic observatories (shown in figure 2). In case of changes in observatory locations and measurement discontinuities (jumps), were it occurred, and reported in the hourly mean measurements, they are taken into account by adjusting the geomagnetic measurements to the level of the most recent epoch. For more on geomagnetic observatory measurements, refer to Matzka et al. (2010) for a more complete description of observatory data and the signals they contained. Also, measurements based on the RC index produced from 21 worldwide observatory locations and for the same time span as for the geomagnetic observatory measurements are also used in this study.

\subsection{Model}

Since the study involve description of the external fields, our requirement for modelling the field contributions is to use a model that will give a detailed description of the external fields particularly that can be used to separate the rapidly varying external field from the internal field. There are several models available that detail the description of the external fields. The POMME model series (Maus et al. 2010, version six) incorporating a dedicated magnetospheric field model (Luhr and Maus, 2010) that requires five solar-terrestrial systems parameters as input is one such model. The GRIMM-3.2 field model covering 2001-2010 is another. Although it does not aim to describe distinct external field sources, but its whole range of vector CHAMP measurements is used to separate the rapidly varying external field from the internal field, with its external field parameterization closely following that of the IGRF-12 candidate model (Lesur et al. 2015, Thomas and Lesur, 2007). Other models includes the CHAOS model (Olsen et al. 2014), and the Comprehensive Models (Sabaka et al. 2004).

In this study, we make use of the CM4 model. There is a new version of the CM4 (Sabaka et al. 2015, version five) model. We decide not to use it in order to preserve the independence of the RC index, which is used in the CM5. In the next section, we briefly describe the CM4 model and justify our decision to use it. 


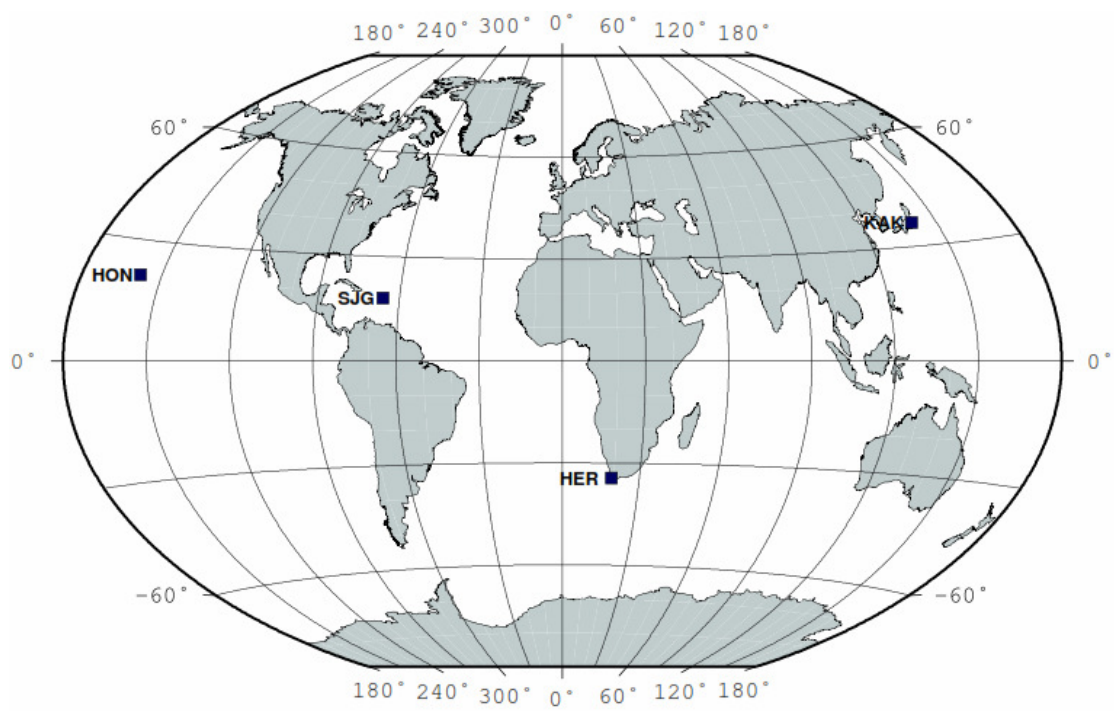

Figure 1: Worldwide locations of the Dst observatories.

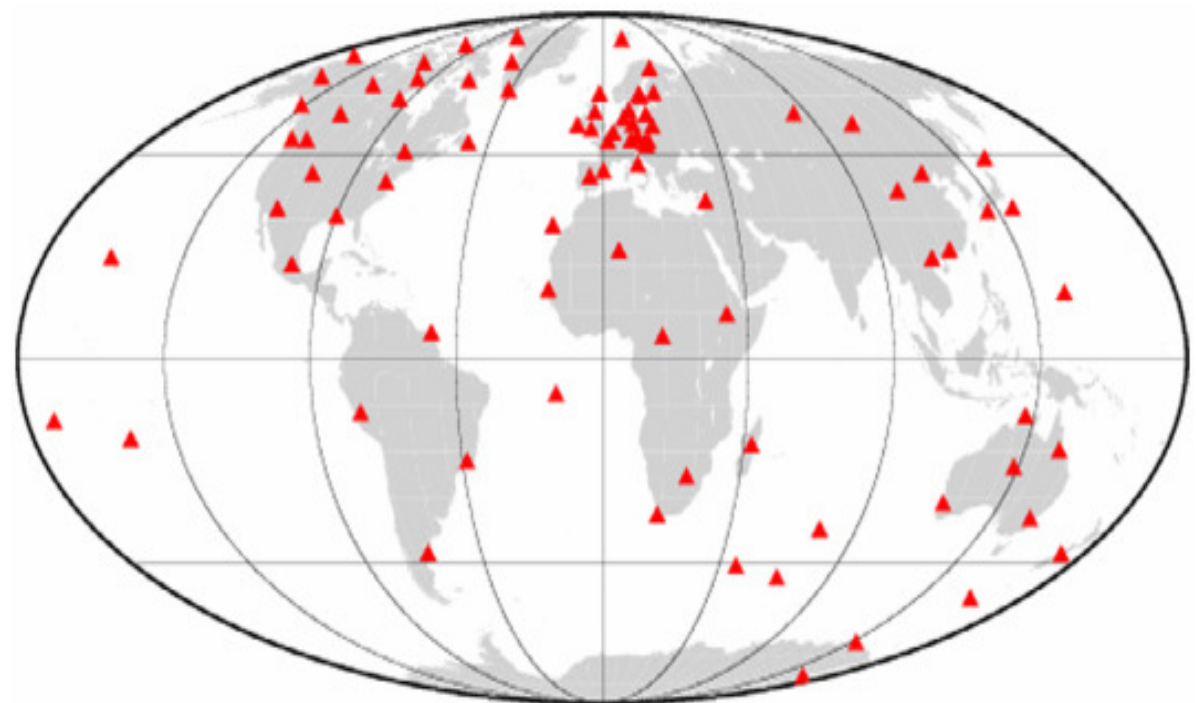

Figure 2: Worldwide spatial distribution of the 109 geomagnetic observatories where measurements were obtained from.

\subsubsection{Comprehensive Model (CM4)}

The comprehensive model, version four (CM4) is used extensively in this study as a comparison to the observatory measurements. CM4 developed by Sabaka et al. (2004) spanning 1960-2002, and following the 'comprehensive approach' estimates all internal and external sources of the geomagnetic field in a joint inversion of OHMs and satellite measurements. The CM4 model is not intended as a true predictive model in time, but the model generates a standardised model of the geomagnetic field at a given location based on prior measurement i.e. the predictive capabilities of the CM4 model is limited to prediction of expected values in areas without measurement coverage based on the available measurement at other locations. The measurements the CM4 model uses to produce its expected 'prediction' are derived from both geomagnetic observatories and different satellite missions i.e. Magsat, POGO, Orsted and CHAMP. The measurements includes vector as well as scalar measurements, in addition to Dst and F10.7, and behavioural knowledge from satellite sampling of $\mathrm{F}$ - region current-induced fields. The CM4 model is capable of modelling some of the toroidal magnetic fields in the ionosphere, the effect of the ring current in the magnetosphere, and the effect of increased solar flux, which is treated as scaling the ionospheric field without changing its shape. The CM4 code is designed to fit solar quiet, Sq periods, and data from more disturbed periods are not, in general, incorporated into the model parameters. The codes come with prewritten driver examples, with the 'example 2' driver code used in this study. This 'example 2' driver code allows the model to output values of induced and external components of the magnetosphere and ionosphere in the $X, Y$, and $Z$ components for a user specified location and time frame (producing a set of six model values) for a given time. Since the CM4 model is constrained by the actual measurements recorded at observatories and during some satellite missions, it is used to gain understanding of how the geomagnetic field behaves over short time scales. 


\section{Indices - Dst and RC}

Dst (Disturbance storm time) was discovered as a signature characteristic of a magnetic storm associated with the ring current by Moos (1910), an intensified current in the magnetosphere which circles the equatorial region (Mendes Jr. et al. 2006). Following the International Geophysical Year (1957-1958), considerable efforts were made to define a global index for the equatorial ring current and in 1969 IAGA endorsed a version of the Dst index as proposed by Sugiura (1969). The Dst index which is based on the horizontal components of the geomagnetic field (as recorded at four near-equatorial geomagnetic observatory stations - see Figure 1) aim to monitor variations of the equatorial magnetospheric ring current. Dst has been known to suffer setbacks when used in geomagnetic modelling, particularly during periods of rapid variations for days away from quiet time. This is as a result of the baseline of Dst which is known to change with time (Olsen et al. 2005; Luhr and Maus 2010), and this has hampered its use in geomagnetic modelling. To enhance its use in geomagnetic field modelling, several improvements have been done on the Dst index, but despite these improvements Dst still suffers from its well-known baseline instabilities and time dependence.

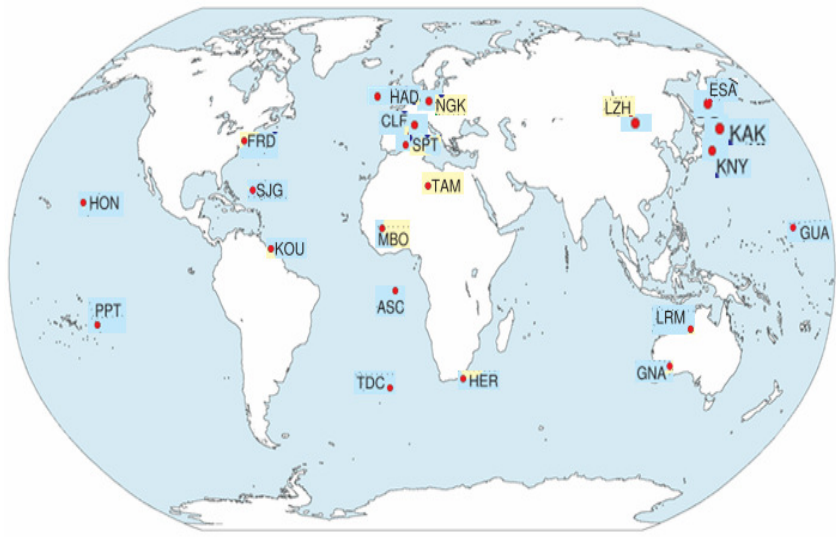

Figure 3: Locations of observatories used for deriving the RC index.

To improve time dependence and describe the strength of the magnetospheric ring current even during geomagnetic quiet conditions, when the baseline instabilities of Dst shows less than optimal results, Olsen (2002) built another type of index - the $\mathrm{RC}$ index (Olsen et al, 2014). The RC index aim (similar to the Dst index) to monitor variations of the equatorial magnetospheric ring current. It is originally derived from OHMs at 21 globally distributed geomagnetic observatory stations (see Figure 3) at low and midlatitudes (excluding locations close to the geomagnetic equator that are affected by equatorial electrojet), focusses on having a stable baseline. According to

Luhr et al. (2017), RC is much more consistent with direct observatories of the ring current effect made by the CHAMP satellite than Dst. As a result it has replaced Dst in current geomagnetic field models i.e. the Swarm Initial Field Model (Olsen et al. 2015) as an hourly proxy for the magnetospheric field intensity.

Figure 4 gives a comparison of the RC index and Dst index from Kauristie et al. 2016. While their morphology is generally similar, some differences can be observed. A small offset is noticeable, particularly at quiet time period, between the RC and Dst indices. While the day to day disturbance are similar, their amplitudes sometimes differ. This may be because RC only considers observatories within limited range of local time, and includes many more selections of observatories than Dst (21 observatories for RC to 4 observatories for Dst). The primary advantage which RC has over Dst is the consistent manner in which secular variation is removed - including fitting detrending polynomials within windows which is known to result in baseline instabilities for the Dst index (Temerin and Li, 2015), is avoided (Kauristie et al. 2016).

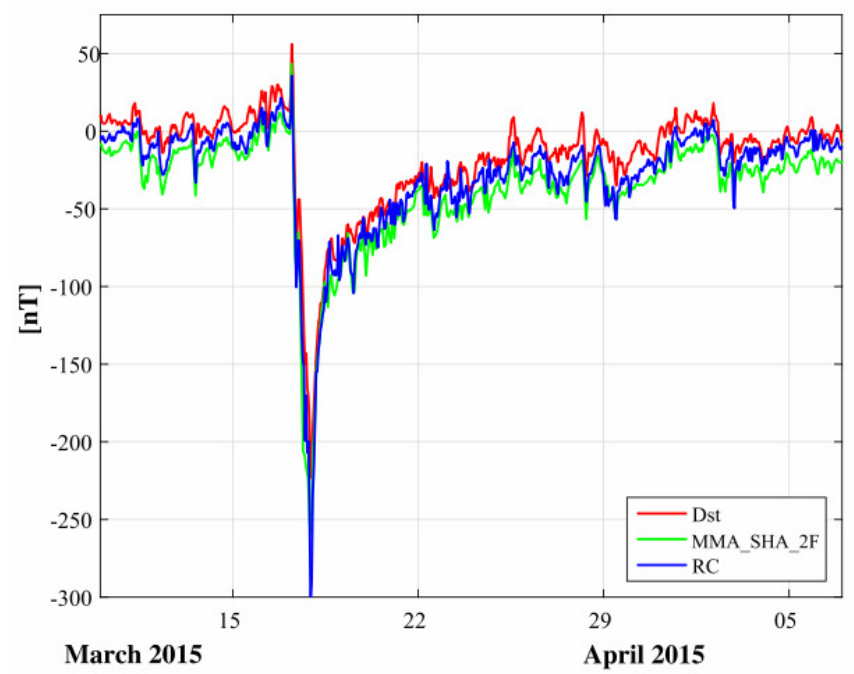

Figure 4: Comparison of ground observatory measurements based on Dst index (Sugiura and kamei, 1991) in red, and the RC index (Olsen et al. 2014), in blue for a month around the occurrence of St, Patrick's day geomagnetic storm on 17th March 2015 (from Kauristie et al. 2016). The green line is the satellite-data based MMA_SHA_2F index (Hamilton 2013) - not applicable to this study.

\section{Methodology}

In this study, we employed the use of the CM4 model as a comparison for our observatory measurements. We have OHMs measurements for days away from quiet time that are showing Sa diurnal variation. On the average, this is well fit by the CM4 model. When compared with individual days show some deviations. These deviations are of two kinds:

- Long period (a day or so) variations that show variations between days of slowly varying signals i.e. difference in Sq signal from the average, or long time unmodelled magnetosphere/induction?

- More rapid variations, particularly seen on our noisier measurements. This is where our focus lies, as this is where the CM4 model finds it difficult to reasonably fit the OHMs measurements for days away from quiet time. So, this is looked at against the RC index.

since our focus is looking at OHMs measurements outside the CM4 operational period (1960-2002.5), by adding additional data, recent Dst and F10.7 measurements and a modification of the CM4 code to extend the operational time series, allows us to extend the CM4 model, particularly for external and induced fields. Our interest is on the external variation fields and the geomagnetic field as used typically in exploration 
industry surveys, so the parts of the CM4 which are not designed to be extended (i.e. the core and lithospheric generated fields) are not so important. The rapid variations of the geomagnetic field are more useful to understand in the situation of a survey, since they constitute the part that would be affected most by any applied corrections, rather than the total field strength.

In this study, we use the penalised least-squares technique with spaced time cubic B-spline based on DeBoor (1978) algorithm. This is to enable us analyse the ionospheric and magnetospheric signals in our geomagnetic diurnal variation observatory measurement, for which we found it necessary to remove some trends that are associated with these signals and look at what is left (the residuals). The residuals consist of unmodelled signals found by subtracting values predicted by the model (comprehensive model in our case) from the observatory data. This is done by detrending the data sequences with spline fits, and we use the spline to fit a spline curve through the CM4 predictions, predictions of this fit are then needed for higher density times given by the observatory data. The CM4 model is used to generate all the synthetic measurements and predictions for both the ionospheric and magnetospheric fields for particular observatory (day and period). We then obtain the measurement that remove these predictions so that we can look at the eigenvalues and eigenvectors of what is left over (the residuals), in order to not only see to what extent CM4 can make useful predictions, but to also study the field that is leftover.

For our Sq variation observatory measurements showing more rapid variation, we look for this at detrending the data sequences with spline fits, and compare these data with the RC index measurements. It was possible to compare individual trends against RC measurements, however, possibly more interesting to compare an eigenvector decomposition of the residuals from the detrending. Expectation might be that the noisiest of the three eigenvectors (i.e. largest eigenvalue) would correlate particularly well with RC index variations. For these RC variations, it might be particularly interesting to look at the decomposition of two combined observatories. If these two combined observatories show large variations that are coherent, then they would show up in combination in the eigenvector for the largest eigenvalue, and this eigenvector could then be compared against the RC index variations. Comparing multiple observatories at one time is particularly a question to see whether RC was doing well for all observatories globally, and whether the RC index is a good representation of all observatories for rapid variation. If it is a good representation, then this suggests that the rapid variations are coming from a large scale source, and therefore corrections for disturbed times (e.g. exploration corrections) may be considered to be global, and looking at rapid observatory results combined (more than one base station) might be a good remote referencing technique.

\section{Results and Discussion}

The CM4 model is used to generate all the synthetic data. In doing this certain part of the model was modified to subtract specific field contributions in order to generate measurements of interest:

- Removing the contributions from ionosphere and magnetosphere i.e. measurement uncorrected with CM4.

- Including contributions from ionosphere and magnetosphere i.e. measurement corrected with CM4.

- Including contributions from ionosphere only i.e. measurement corrected for ionosphere only (excluding contributions from magnetosphere).

- Including contributions from magnetosphere only i.e. measurement corrected for magnetosphere only (excluding contributions from ionosphere).

Here, we present the results and discussion of the treatment of the covariance by considering the covariance between the diurnal variation residuals in the $X, Y$, and $Z$ directions for each observatory. Here, we look at the case of combined observatories, as we are interested in looking at the decomposition for two combined observatory station measurements at a time; to look at the covariance in the behaviour of the three components for the residual measurements for the different field contributions specified above. We calculated the eigenvalues and eigenvectors from a $6 \times 6$ covariance matrix. In the $6 \times 6$ case, we obtained 6 eigenvalues and 6 eigenvectors, with the first 3 eigenvectors $\left(v_{1}, v_{2}, v_{3}\right)$ representing that of the first observatory and the next 3 eigenvectors $\left(v_{4}, v_{5}\right.$, and $\left.v_{6}\right)$ representing that of the second observatory. This corresponds to each eigenvalue $(\lambda)$, with the eigenvalue with the largest value being the one of interest - as it's the one with the noisiest signal. It is presumed that if these two combined observatories show large variations that are coherent, then the expectation is that they would show up in combination in the eigenvector for the largest eigenvalue.

Hence, we computed iteratively the eigenvalues and eigenvectors from the covariance matrix of the residuals for the combined observatories. This is achieved using full daily residual OHMs measurements with applied corrections, where needed, to the input measurement calculated using the CM4 model. The use of the CM4 model to correct the input measurements before calculating the eigenvalues and eigenvectors removes a strong bias from internal field sources. The final eigenvalues and eigenvectors for selected two combined European observatories (Niemegk, NGK and La Aquila, AQU) and a selected combined European and non-European observatories (Niemegk, NGK and Mbour, MBO) are shown in tables 1 and 2. For each case we show the eigenvalues and eigenvectors calculated for case when the measurement residuals is corrected with CM4 (contributions from ionosphere and magnetosphere computed), and case when measurement residuals is uncorrected with CM4 i.e. raw data (no contribution from ionosphere and magnetosphere computed). 
Table 1: Final eigenvalue and eigenvector for combined European observatory station measurements (NGK and AQU) with (a) showing values for measurements corrected with CM4 i.e. contributions from ionosphere and magnetosphere included and (b) showing values for measurements uncorrected with CM4 i.e. raw data, with contributions from ionosphere and magnetosphere excluded. They are mean values for all the disturbed days (Kp $\leq 5)$ in May, 2006.

\begin{tabular}{|l|l|l|l|l|l|l|l|}
\hline $\mathrm{S} / \mathrm{N}$ & $\lambda$ & $\mathrm{V}_{1}$ & $\mathrm{~V}_{2}$ & $\mathrm{~V}_{3}$ & $\mathrm{~V}_{4}$ & $\mathrm{~V}_{5}$ & $\mathrm{~V}_{6}$ \\
\hline 1 & 217.22 & 0.730 & -0.161 & 0.029 & 0.635 & -0.193 & 0.027 \\
\hline 2 & 94.57 & 0.222 & 0.773 & 0.014 & 0.120 & 0.579 & -0.060 \\
\hline 3 & 6.27 & -0.616 & 0.773 & 0.259 & 0.710 & -0.058 & -0.195 \\
\hline 4 & 5.28 & 0.118 & -0.119 & 0.946 & -0.172 & 0.144 & 0.162 \\
\hline 5 & 2.12 & -0.144 & -0.346 & -0.187 & 0.222 & 0.567 & 0.690 \\
\hline 6 & 0.99 & 0.069 & -0.484 & -0.039 & -0.004 & 0.552 & -0.674 \\
\hline
\end{tabular}

(a)

\begin{tabular}{|l|l|l|l|l|l|l|c|}
\hline $\mathrm{S} / \mathrm{N}$ & \multicolumn{1}{|c|}{$\lambda$} & \multicolumn{1}{|c|}{$\mathrm{V}_{1}$} & \multicolumn{1}{|c|}{$\mathrm{V}_{2}$} & \multicolumn{1}{|c|}{$\mathrm{V}_{3}$} & \multicolumn{1}{|c|}{$\mathrm{V}_{4}$} & $\mathrm{~V}_{5}$ & $\mathrm{~V}_{6}$ \\
\hline 1 & 785.54 & -0.103 & 0.697 & 0.103 & -0.259 & 0.635 & 0.148 \\
\hline 2 & 323.52 & 0.825 & 0.133 & 0.254 & 0.428 & 0.069 & 0.223 \\
\hline 3 & 37.45 & -0.019 & -0.250 & 0.722 & -0.494 & -0.138 & 0.391 \\
\hline 4 & 3.71 & -0.031 & -0.545 & -0.335 & 0.060 & 0.546 & 0.537 \\
\hline 5 & 3.51 & -0.523 & -0.016 & 0.468 & 0.698 & 0.136 & 0.029 \\
\hline 6 & 1.41 & -0.185 & 0.368 & -0.269 & 0.120 & -0.506 & 0.698 \\
\hline
\end{tabular}

(b)

Table 2: Final eigenvalues and eigenvectors for combined European and non-European (African) observatory station measurements (NGK and $\mathrm{MBO}$ ) with (a) showing values for measurements corrected with CM4 i.e. contributions from ionosphere and magnetosphere included, and (b) showing values for measurements uncorrected with CM4 i.e. raw data, with contributions from ionosphere and magnetosphere excluded. Same period as in table 1

\begin{tabular}{|l|l|l|l|l|l|l|l|}
\hline $\mathrm{S} / \mathrm{N}$ & $\lambda$ & $\mathrm{V}_{1}$ & $\mathrm{~V}_{2}$ & $\mathrm{~V}_{3}$ & $\mathrm{~V}_{4}$ & $\mathrm{~V}_{5}$ & $\mathrm{~V}_{6}$ \\
\hline 1 & 202.14 & 0.743 & -0.193 & 0.034 & 0.509 & -0.344 & -0.179 \\
\hline 2 & 92.55 & 0.280 & 0.703 & 0.036 & 0.193 & -0.343 & 0.520 \\
\hline 3 & 23.04 & 0.200 & 0.598 & -0.149 & 0.178 & 0.583 & -0.456 \\
\hline 4 & 11.57 & 0.370 & -0.292 & -0.106 & -0.005 & 0.626 & 0.613 \\
\hline 5 & 7.86 & -0.403 & 0.158 & 0.317 & 0.781 & 0.064 & 0.313 \\
\hline 6 & 4.21 & 0.173 & -0.011 & 0.929 & -0.249 & 0.169 & -0.124 \\
\hline
\end{tabular}

(a)

\begin{tabular}{|l|l|l|l|l|l|l|l|}
\hline $\mathrm{S} / \mathrm{N}$ & \multicolumn{1}{|c|}{$\wedge$} & \multicolumn{1}{|c|}{$\mathrm{V}_{1}$} & \multicolumn{1}{|c|}{$\mathrm{V}_{2}$} & \multicolumn{1}{|c|}{$\mathrm{V}_{3}$} & \multicolumn{1}{|c|}{$\mathrm{V}_{4}$} & \multicolumn{1}{c|}{$\mathrm{V}_{5}$} & $\mathrm{~V}_{6}$ \\
\hline 1 & 832.58 & -0.029 & 0.657 & 0.137 & -0.586 & 0.308 & 0.332 \\
\hline 2 & 314.42 & 0.830 & -0.053 & 0.268 & -0.148 & -0.420 & 0.197 \\
\hline 3 & 56.86 & 0.444 & 0.503 & -0.337 & 0.550 & 0.332 & -0.155 \\
\hline 4 & 19.41 & -0.122 & 0.412 & -0.331 & -0.224 & -0.650 & -0.482 \\
\hline 5 & 4.95 & -0.187 & 0.090 & -0.400 & 0.262 & -0.366 & 0.771 \\
\hline 6 & 4.01 & -0.253 & 0.367 & 0.726 & 0.462 & -0.247 & -0.002 \\
\hline
\end{tabular}

(b)
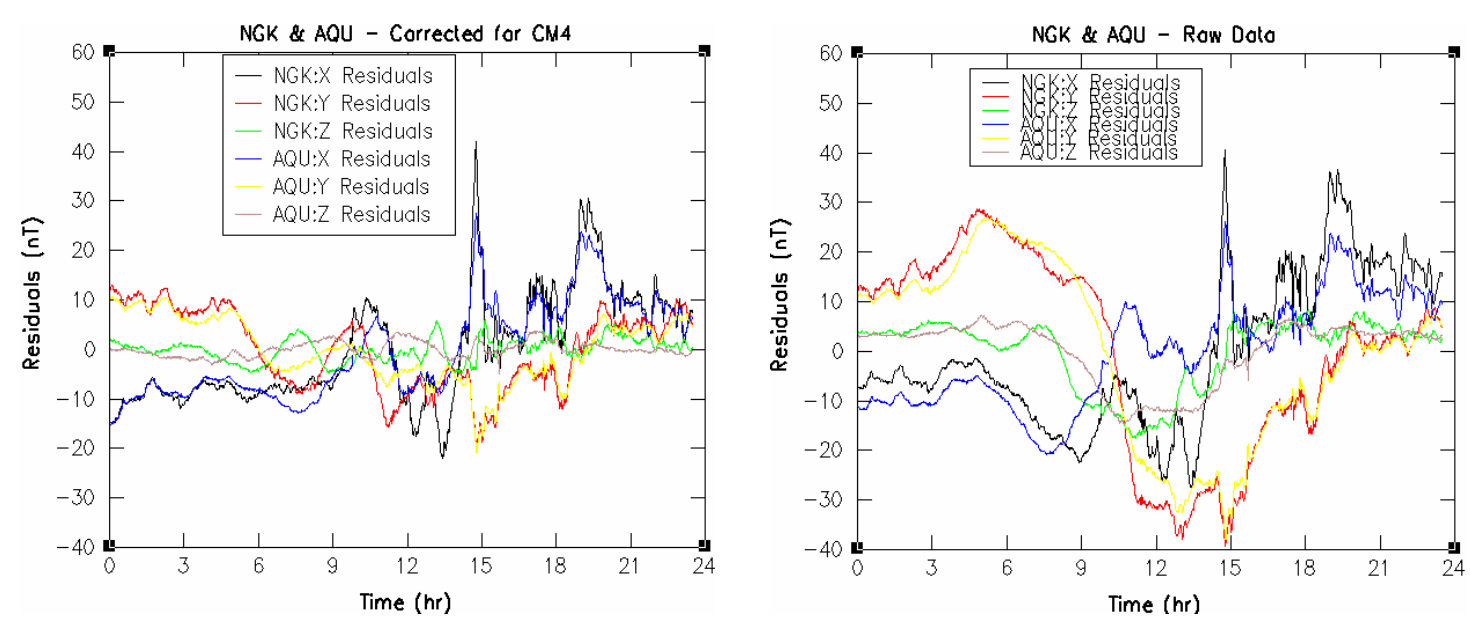

Figure 5: Plots of the $\mathrm{X}, \mathrm{Y}$, and $\mathrm{Z}$ components derived from residual measurements used in calculating the eigenvalues and eigenvectors for combined European observatories (NGK and AQU) for measurements corrected with CM4 (left) and measurements uncorrected with CM4 i.e. raw data (right), for month May, 2006. 

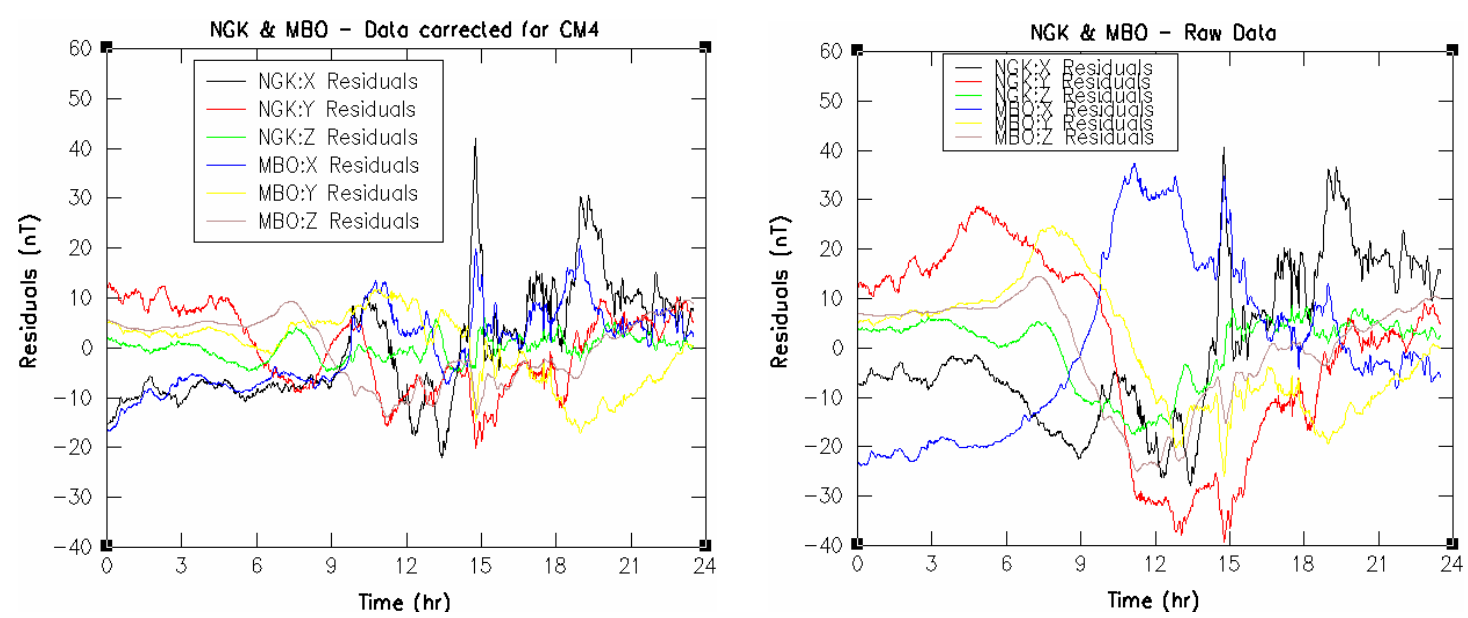

Figure 6: Plots of the $\mathrm{X}, \mathrm{Y}$, and $\mathrm{Z}$ components derived from residual measurements used in calculating the eigenvalues and eigenvectors for combined European observatories (NGK and MBO) for measurements corrected with CM4 (left) and measurements uncorrected with CM4 i.e. raw data (right), for month of May, 2006.

The results of the eigenvalue and eigenvector for both combined European observatories and combined European and non-European observatories show that the noisy signals get combined in the two vectors with the two largest eigenvalues. The largest eigenvalues in all the combined observatories studied correlates very well i.e. the $\mathrm{X}, \mathrm{Y}$, and $\mathrm{Z}$ components from both observatories show good correlation. This is easily observed by just looking at the measurement residual plots (Figures 5 and 6 ) produced from the eigenvalues and eigenvectors result for both cases shown in tables 1 and 2 above. They correlate reasonably well.

In the combined European observatories, the covariance comes between the $X$ and $Y$ components, as the eigenvectors $V_{1}$ and $V_{2}$, and $V_{4}$ and $V_{5}$ seen in both cases specified in Table 1. Also, we can observe that most of the covariance come between the $X$ and $Y$ components in Table 2 for the case where the residual measurements is corrected with $\mathrm{CM} 4$, but we can also see some $Z$ component covariance with the $X$ component where the residual measurement is uncorrected with CM4 i.e. raw data.

\subsection{Comparing with the $R C$ index.}

Next we compare the RC index residuals against the OHMs measurement residuals i.e. we compare the eigenvector decomposition of the OHMs measurement residuals from our detrending for both cases, corrected and uncorrected with CM4. The RC index residual was obtained by subtracting Dst index values from that of the RC values (in order words, we removed a Dst trend to look at the small temporal scale ring current variation after the subtraction of Dst). This was analogous to what we did to obtain the observatory data residuals (subtracting the model predictions from the observatory station data). Expectation is that the noisiest of the three eigenvectors i.e. eigenvalue, would correlate very well with the RC index variations for our combined observatory measurements. So, we compare the RC index residuals with the combined observatory measurement residuals, focussing on the $\mathrm{X}$ component residual measurement of our observatory measurement. This is because the $\mathrm{X}$ component of our observatory measurements is the component that is most influenced or affected by the external field sources of the geomagnetic diurnal variation field. Our expectation is that (assuming we are correct) the largest eigenvalue, which is consistently the $X$ component, from our covariance analysis result would correlate well with the RC index residual variations.

As seen in figures 5 and 6 , our combined observatory measurements show large variations which are coherent when the CM4 model corrections were applied. This is observed in both the combined European observatories and the combined European and non-European observatories, and is even seen in combined non-European observatories (not shown) studied. This is revealed in the combination of the eigenvector for the largest eigenvalue.

Figures 7, 8, and 9 show the plots for the comparison between the $\mathrm{X}$ component measurements of some selected combined observatories and the RC index residuals. Figures 7 and 8 are for combined observatories used in calculating the RC index, while figure 9 involve combined observatories that were not involved in calculating the RC index. From the plots we can observe that clearly $R C$ is doing a good job fitting the fine and not so fine variations of the signals, particularly when corrected with CM4, irrespective of whether measurements from the observatories are involved in deriving the RC index or not. Also, the correlation is better for the combined European observatories (NGK \& AQU) (figure 7), than for the combined European and non-European observatories (figure 8).

In the case of the combined European and nonEuropean observatories not involved in the calculation of the RC index (BDV \& BNG) in figure 9, we can also observe the $X$ component measurement residuals correlating well with the RC index residuals. We can observe similar patterns as seen in figures 7 and 8 . In all, we see that there is a better agreement with RC index residuals in cases where the $\mathrm{X}$ component measurement residuals are corrected with the CM4 model for both ionosphere and magnetosphere, and when corrected for ionosphere only, compared with cases when no CM4 correction is done (raw data) and when corrected for magnetosphere only, respectively. 

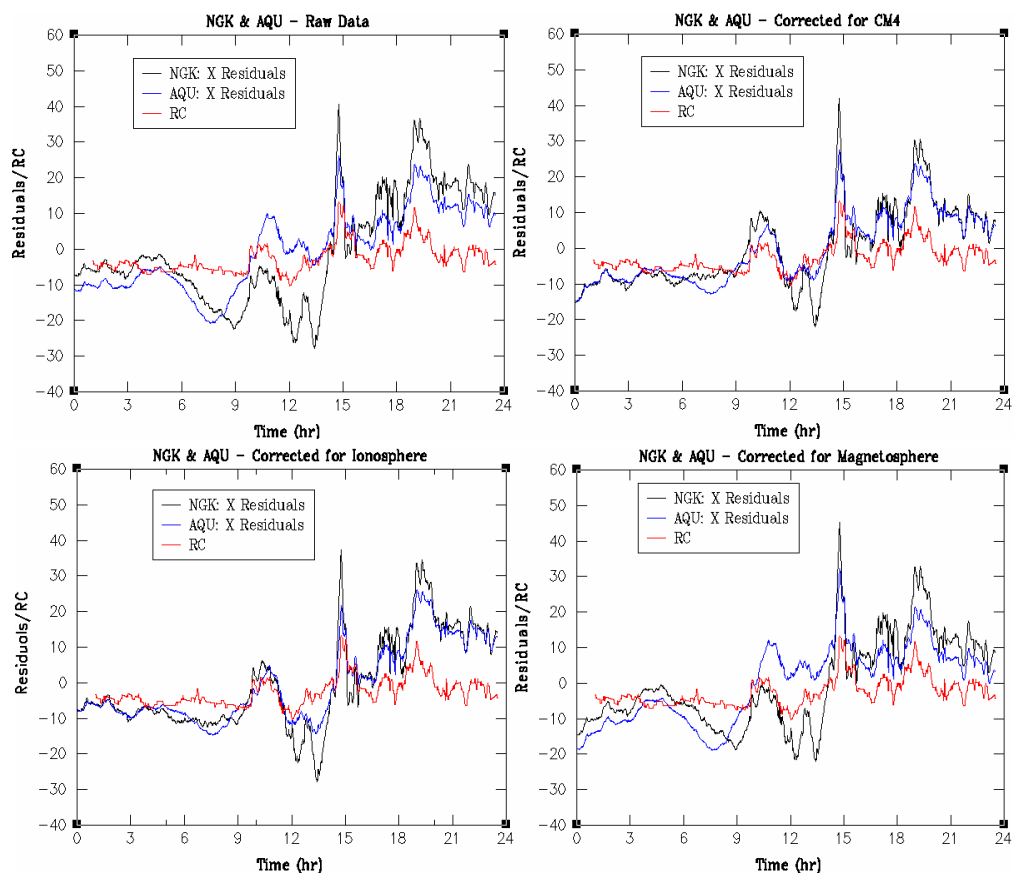

Figure 7: Combined European observatories (NGK \&AQU) and RC index comparison for $30^{\text {th }}$ May 2006 for the different field contributions.
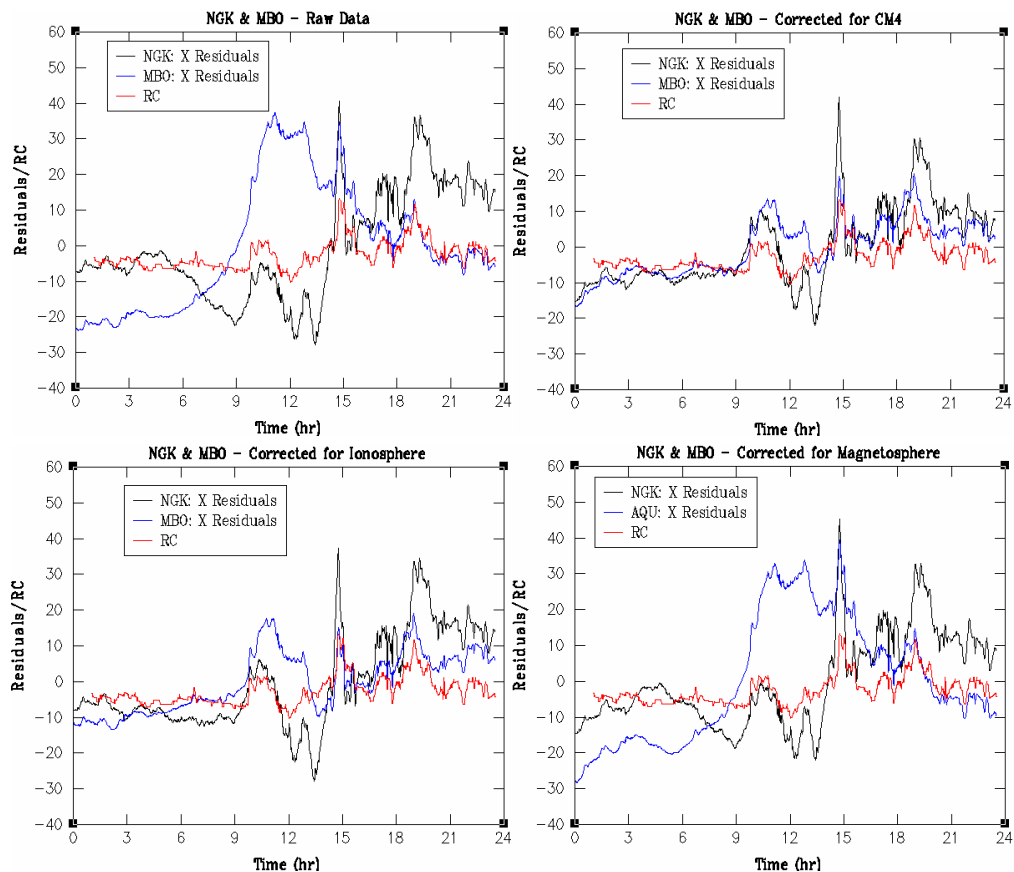

Figure 8: Combined European and non-European (African) observatories (NGK \& MBO) and the RC index comparison for $30^{\text {th }}$ May 2006 for the different field contributions.

The ionosphere clearly does a good job of removal compared to the magnetosphere which obviously doesn't get rid of the rapid variations, because Dst (as used in the CM4 model) is not high enough time resolution.

\subsection{Correlation between Observatories and $R C$ index Residuals}

Encouraged by the good agreement and coherence seen in the plots comparing the RC index against the $X$ component residuals of our combined geomagnetic observatory measurements, and to further confirm this the residuals of the $X$ component measurements and the RC index are further analysed by means of cross-correlation coefficient. We performed a cross-correlation between the combined observatory $X$ component and the RC index residuals. We also performed a cross-correlation between the $X$ component residuals of our combined observatories in different locations, both within the same region and different regions geographically. The results of this cross-correlation coefficients operation is shown in Tables 3 and 4. 

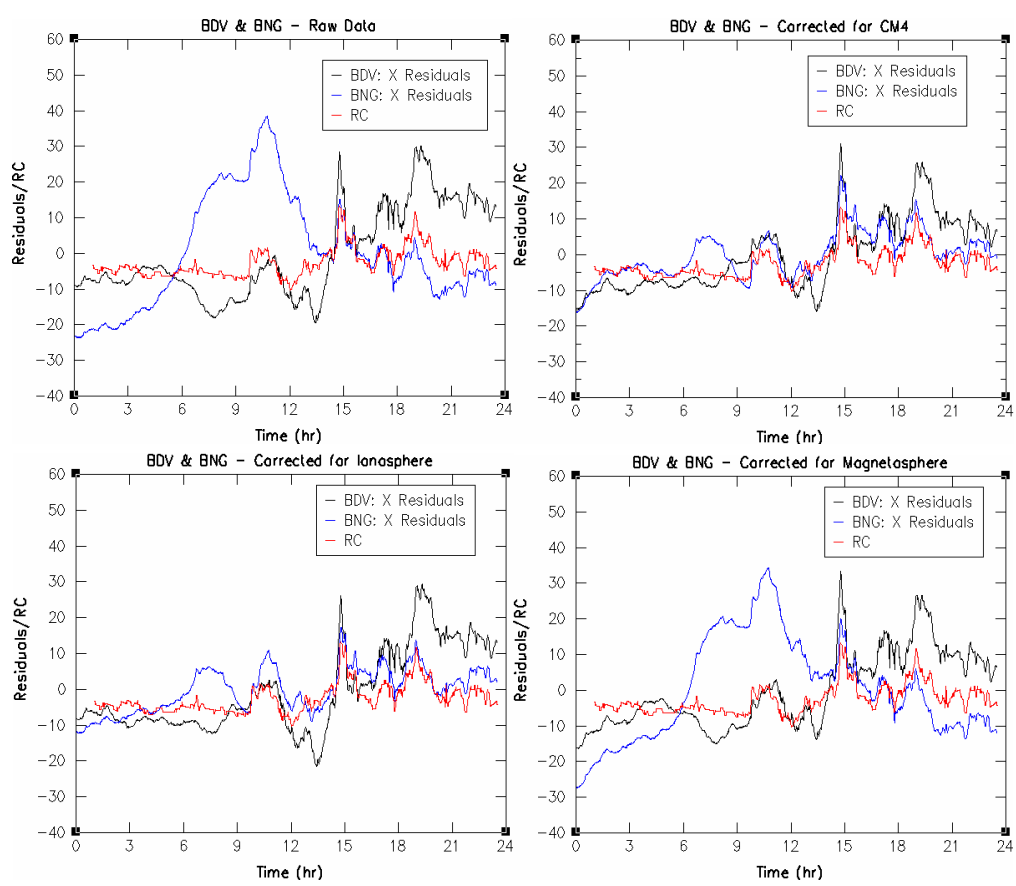

Figure 9: Combined European and non-European observatories (BDV \& BNG) and the RC index comparison for $30^{\text {th }}$ May 2006. Unlike figures 7 and 8 above, none of the combined observatories here are part of the RC observatories i.e. observatories where measurements are used for deriving the RC index.

Table 3: Cross-correlation coefficients of $X$ component residuals of selected combined observatories with the $\mathrm{RC}$ index located within the same geographic region and in different geographic regions, for 30th May 2006.

\begin{tabular}{|c|c|c|}
\hline $\begin{array}{c}\text { Combined } \\
\text { Observatory }\end{array}$ & Code & $\begin{array}{c}\text { Cross- } \\
\text { correlation } \\
\text { coefficient } \\
\text { X comp. with } \\
\text { RC index }\end{array}$ \\
\hline Niemegk \& L'Aquila & NGK-AQU & 0.90 \\
\hline Niemegk \& Bangui & NGK-BNG & 0.80 \\
\hline L'Aquila \& Mbour & AQU-MBO & 0.80 \\
\hline Alma Ata \& Mbour & AAA-MBO & 0.80 \\
\hline $\begin{array}{c}\text { Huancayo \& } \\
\text { Addis Ababa }\end{array}$ & HUA-AAE & 0.75 \\
\hline Budkov \& Kakadu & BDV-KDU & 0.75 \\
\hline Vassouras \& Del Rio & VSS-DLR & 0.70 \\
\hline Gnangara \& Kakadu & GNA-KDU & 0.70 \\
\hline Bangui \& Mbour & BNG-MBO & 0.90 \\
\hline Boulder \& Del Rio & BOU-DLR & 0.85 \\
\hline $\begin{array}{c}\text { PhuThuy \& } \\
\text { Beijing Ming Tombs }\end{array}$ & PHU-BMT & 0.90 \\
\hline L'Aquila \& Trelew & AQU-TRW & 0.75 \\
\hline Huancayo \& & HUA-VSS & 0.85 \\
\hline Vassouras & HUS & \\
\hline
\end{tabular}

The results of the cross-correlation coefficients between the $X$ component of the combined observatories and that of the RC index confirm the good correlation and agreement seen in the various plots in figures 7, 8, and 9. A very good crosscorrelation coefficients of between 0.70 and 0.90 can be seen to exist between the $X$ component of our combined observatories and the RC index residuals. The results of the cross-correlation coefficients did not show any geographical discrimination or dependence as we see high cross-correlation coefficients for our combined observatories both between European, and between the European and non-European observatories with the RC index residuals. Even our combined non-European observatories with the RC index show high cross-correlation coefficients between the $X$ component measurements and the $\mathrm{RC}$ index residuals, as seen in the table above. Comparing the $X$ component measurement residuals for the combined non-European observatories and the RC index residuals was particularly a question to see whether RC was doing well for all (or most) observatories globally or only the European ones.

Table 4: Cross-correlation coefficients of $X$ component between observatory measurements located within the same and different geographical locations. Notice the high cross-correlation between the $\mathrm{X}$ components of the field at different locations irrespective of geographical region, for 30th May 2006.

\begin{tabular}{|c|c|c|}
\hline $\begin{array}{c}\text { Combined } \\
\text { Observatory }\end{array}$ & Code & $\begin{array}{c}\text { Cross- } \\
\text { correlation } \\
\text { coeffi. of } \mathbf{X} \\
\text { residuals } \\
\text { between } \\
\text { observatories }\end{array}$ \\
\hline Niemegk \& L'Aquila & NGK-AQU & 0.90 \\
\hline Bangui \& Mbour & BNG-MBO & 0.90 \\
\hline $\begin{array}{c}\text { Phu Thuy \& } \\
\text { Beijing Ming Tombs }\end{array}$ & PHU-BMT & 0.95 \\
\hline Gnangara \& Kakadu & GNA-KDU & 0.90 \\
\hline Boulder \& Del Rio & BOU-DLR & 0.85 \\
\hline Vassouras \& Trelew & VSS-TRW & 0.80 \\
\hline Niemegk \& Bangui & NGK-BNG & 0.95 \\
\hline Alma Ata \& Mbour & AAA-MBO & 0.85 \\
\hline Kakadu \& Budkov & KDU-BDV & 0.80 \\
\hline $\begin{array}{c}\text { Huancayo \& } \\
\text { Addis Ababa }\end{array}$ & HUA-AAE & 0.80 \\
\hline Vassouras \& Del Rio & VSS-DLR & 0.70 \\
\hline L'Aquila \& Trelew & AQU-TRW & 0.70 \\
\hline L'Aquila \& Mbour & AQU-MBO & 0.85 \\
\hline
\end{tabular}


The result of the cross-correlation coefficients also confirm that the selected combined observatories show high cross-correlation coefficient between the $X$ component residuals of the field. This is particularly high for observatories located within the same geographical regions i.e. NGK \& AQU (0.90), BNG \& MBO (0.90), PHU \& BMT (0.95), etc. That of observatories located at different geographical regions also show high cross-correlation coefficients as shown in Table 4. The high cross-correlation coefficient exhibited by the signals of the $X$ component of the combined observatory measurements between themselves, and with the RC index makes us to assume that the differences in local features or regional features may not have affected the signals, at least as far as their time variation is concerned, otherwise cross-correlation coefficient between their residuals would have been expected to vary with location more characteristically. Hence, we can say that given the magnitude of the cross-correlation coefficient that it is independent of region and there suggest that the rapid variation seen in our OHMs measurements are coming from a large scale source, possibly ring current magnetosphere, external to the Earth.

\section{Conclusion}

In this study we have looked at the error correlation of our observatory measurement field model residuals by taking the approach of calculating the covariance directly from the residuals. The results show that much of the covariance comes between the residuals of the $X$ and $Y$ components of our observatory measurements. This is unlike what has been generally observed in slow and longer variations (seen in single observatories), where it is observed that strong correlation is between the $\mathrm{X}$ and $\mathrm{Z}$ components of the observatory measurement residuals.

In considering the eigenanalysis of our combined days, the comparison of the field component residuals with the RC index is doing a reasonable good job fitting the fine variations of the signals. This is particularly seen in the $X$ component residual measurements. This is not overly surprising, as this is the field component most influenced and affected by external field sources. Hence, we conclude that the fine variations are global or has global phenomenon as it is observed in all the observatories studied globally. When the ionosphere and magnetosphere field is taken away from the measurements through CM4 corrections, the ionosphere is seen to do a good job of removal, but the magnetosphere doesn't get rid of the fluctuations observed in our measurements, as Dst is not high enough time resolution.

Our results show clearly that some external influences are dominating our observatory residual measurement variations. This may suggest evidence of the presence of magnetospheric ring current causing the fluctuations/rapid variations observed in our observatory measurement plots. This is further amplified by the good correlation, physically, observed in the comparison of the $X$ component residual measurements and the RC index. To further establish this link between the rapid variations seen in our measurements and the RC index, we measured the cross-correlation coefficients between the $X$ component of our combined observatory measurement and the RC index, and also that between the $X$ components of our combined observatories. The result show, generally, a very high cross-correlation coefficients between the $\mathrm{RC}$ index and the $X$ component of our combined observatory residuals, and also between the $X$ component residuals of the field. These cross-correlation coefficient ranges from 0.70 to 0.95 . The result of this study suggests that the rapid variations seen in our observatory measurements are coming from a large-scale, possibly magnetospheric ring current, and that the RC index is a good representation for rapid variations for most observatories globally, not just European ones. Also, corrections for disturbed times in magnetic explorations may be considered to be global, while observatory results combined i.e. more than a single base station, might be a good remote referencing technique.

\section{Acknowledgement}

The author sincerely thanked Prof. Richard Holme, his PhD supervisor at the University of Liverpool, for all the useful advice in the past. Also, appreciation goes to the management and staff of INTERMAGNET organisation, and all the institutions and staff that operate the geomagnetic observatories, and host the CM4 field model and activity indices (Dst and RC). The author also appreciate the constructive comments from anonymous reviewers.

The study was carried out as part of the author's postgraduate research studies at the University of Liverpool, and funded by the Tertiary Education Trust Fund (TETFUND), Nigeria. 


\section{References}

de Boor, C., 1978. A Practical Guide to Splines, Springer-Verlag, New York.

Hamilton, B., 2013. Rapid modelling of the large-scale magnetospheric field from Swarm satellite data. Earth Planets Space, 65, 1295-1308.

Karinen, A., and Mursula, K., 2006. Correcting the Dst index: Consequences for absolute level and corrections, Journal of Geophys. Res., vol. 111, A08207, doi:1029/2005JA011299.

Kauristie, K., Morschhauser, A., Olsen, N., Finlay, C.C., Mcpheron, R.L., Gjerloev, J.W., and Opgenoorth, H.J., 2016. On the usage of geomagnetic indices for data selection in internal field modelling, Space Sci. Rev., doi: 10.1007/s 11214-0160301-0.

Langel, R.A. \& Estes, R.H., 1985. Large-scale, near-field magnetic fields from external sources and the corresponding induced internal field, J. geophys. Res., 90(B3), 2487-2494.

Lesur, V., Rother, M., Wardinski, I., Schachtschneider, R. Hamoudi, M. \& Chambodut, A., 2015. Parent magnetic field models for the IGRF-12 GFZ-candidates, Earth Planets Space, 67(1), 87-102.

Luhr, H. \& Maus, S., 2010. Solar cycle dependence of quiet-time magnetospheric currents and a model of their near-Earth magnetic fields, Earth Planets Space, 62(10), 843-848.

Luhr, H., Xiong, C., Olsen, N. \& Le, G., 2017. Near-Earth magnetic field effects of large-scale magnetospheric currents, Space Sci. Rev., 206, 521-545.

Matzka, J., Chulliat,A., Mandea, M., Finlay,C.C., and Qamili, E., 2010. Geomagnetic observations for main field studies: From ground to space, Space Science Review, 155, 29-64.

Maus, S., and Weidelt, P., 2004. Separating the magnetospheric disturbance magnetic field into external and transient internal contributions using a 1D conductivity model of the Earth, Geophysical Research Letters, 31, L12, 614.

Maus, S., Manoj, C., Rauberg, J., Michaelis, I. \& Luhr, H., 2010. NOAA/NGDC candidate models for the 11th generation International Geomagnetic Reference Field and the concurrent release of the 6th generation POMME magnetic model, Earth Planets Space, 62(10), 729-735.

Mendes Jr., O., da Costa, A.M., and Bertoni, F.C.P., 2006. Effects of the number of stations and time resolution on Dst derivation, J. Atmos. Sol.-Terr. Phys., 68, 2127-2137.
Moos, N. A. F., 1910. Colaba Magnetic Data, 1846 to 1905. 2. The Phenomenon and its Discussion, Bombay, India: Central Government Press.

Mursula, K. \& Karinen, A., 2005. Explaining and correcting the excessive semiannual variation in the Dst index, Geophys. Res. Lett., 32(14), 1-4.

Mursula, K., Holappa, L. \&Karinen, A., 2011. Uneven weighting of stations in the Dst index, J. Atmos. Sol.-Terr. Phys., 73(2-3), 316-322.

Olsen, N., 2002. A model of the geomagnetic field and its secular variation for epoch 2000 estimated from Ørsted data, Geophysical Journal International, 149, 454-462.

Olsen, N., Sabaka, T.J. \& Lowes, F., 2005. New parameterization of external and induced fields in geomagnetic field modeling, and a candidate model for IGRF 2005, Earth Planets Space, 57(12), 1141-1149.

Olsen, N., Glassmeier, K.-H. \& Jia, X., 2010. Separation of the magnetic field into external and internal parts, Space Sci. Rev., 152(1), 135-157.

Olsen, N., Luhr, H., Finlay, C.C., Sabaka, T.J., Michaelis, I., Rauberg, J. \& Tøffner-Clausen, L., 2014. The CHAOS-4 geomagnetic field model, Geophys. J. Int., 197(2), 815-827.

Olsen, N. et al., 2015. The Swarm Initial Field Model for the 2014 geomagnetic field, Geophys. Res. Lett., 42(4), 1092-1098.

Sabaka, T.J, Olsen, N. and Purucker, M.E., 2004. Extending Comprehensive models of the Earth's magnetic field with Oersted and CHAMP data. Geophysical Journal International, 159, 521-547.

Sugiura, M., 1964. Hourly values of equatorial Dst for the IGY, Ann. Int. Geophys. Year, 35, 4-45.

Temerin, M., and Li, X., 2015. The Dst index underestimates the solar cycle variation of geomagnetic activity. J. Geophys. Res. Space Phys. 120(7), 5603-5607.

Thomson, A. W. P., and Lesur, V., 2007. An improved geomagnetic data selection algorithm for global geomagnetic field modelling, Geophysics Journal International, 169, 951-963. 